\title{
Estudio comparativo de resistencia aeróbica y anaeróbica en jugadores de baloncesto en función de la metodología de entrenamiento
}

\section{Comparative study about aerobic and anaerobic endurance for basketball players based on the training methodology}

\author{
Mancha, D., Ibáńez, S.J., Reina, M. y Antúnez, A.
}

Grupo de Optimización del Entrenamiento y Rendimiento Deportivo. Facultad de Ciencias del Deporte. Universidad de Extremadura.

Resumen: El Objetivo de este trabajo es identificar las diferencias en la capacidad aeróbica y anaeróbica de jugadores en período de formación, que son entrenados con metodologías diferentes. Por ello, es necesario conocer como son las demandas fisiológicas y cinemáticas de los entrenamientos. El diseño de esta investigación se puede enmarcar bajo una estrategia manipulativa, dentro de los estudios de naturaleza empírica de tipo cuasiexperimental donde se busca examinar las diferencias entre grupos, Diseńo de Grupos No Equivalentes.

La población del estudio son dos equipos de baloncesto que entrenan bajo diferentes metodologías pero que disponen del mismo tiempo de entrenamiento y material.

En cuanto a los resultados que se obtienen, se encuentran diferencias en la prueba aeróbica y anaeróbica entre los equipos, siendo superior el equipo bajo enfoque comprensivo.

En este caso, se puede afirmar que entrenar bajo una metodología comprensiva provoca mejores resultados tanto en Resistencia Aeróbica como en Anaeróbica.

Palabras Claves: Resistencia Aeróbica, Resistencia Anaeróbica, Metodología Tradicional, Metodología Comprensiva, Baloncesto.
Abstract: The aim of this investigation is identifying the differences between aerobic and anaerobic capacities in players. They are training with different methodologies. Therefore, it is necessary to know how are the physiological and cinematic request of the trainings.

The design of this investigation can be classified as a manipulative strategy. It is inside of studies about empirical nature of cuasiexperimental type, where you can examinate the differences between groups: Design of not equal groups.

The people group of the study are two basketball teams. They train with differents methodologies but they have the same time to practise and the same material too.

Regarding the results, there are some differences between aerobic and anaerobic events of the teams. In this way, it is superior the team with comprehensive methodology.

In this case, you can affirm that training with a comprehensive methodology causes the best results as aerobic and anaerobic endurance.

Keywords: Aerobic Endurance, Anaerobic Endurance, Comprehensive Methodology, Traditional Methodology, Basketball.

\section{Introducción}

En la actualidad, el baloncesto es considerado uno de los deportes mas practicados en el mundo, este hecho ha llevado a la comunidad científica a mostrar su interés a la hora de investigar sobre esta modalidad deportiva. El baloncesto se compone de habilidades técnico- tácticas complejas que tienen una influencia directa en los requerimientos fisiológicos impuestos sobre el jugador durante la competición (Ziv y Lidor., 2009). En la literatura se puede encontrar multitud de estudios que avalan que dicho deporte tiene un carácter híbrido. Esto se debe a que la mayoría del tiempo la energía empleada por los jugadores proviene de la vía aeróbica (Narazaki, Berg, Stergiou y Chen, 2009) y cuya finalidad principal es la de realzar la resintesis de fosfocreatina y aclaramiento de lactato de la actividad muscular (Castagna, Manzi, Impellizeri, Chaouachi, Abdelkum, Ditroilo, 2010); aunque al igual que

Dirección para correspondencia [Correspodence address]: David Mancha Triguero. Grupo de Optimización del Entrenamiento y Rendimiento Deportivo, Universidad de Extremadura. E-mail: dmanchat@alumnos. unex.es en otros deportes colectivos, existen acciones puntuales que son de carácter explosivo que dependen de la vía anaeróbica del deportista (Chaouachi, Brugheli, Chamari, Levin, Abdelkrim, Laurencelle, Castagna, 2009). Consecuentemente, para jugar a un alto nivel se necesita tener un nivel óptimo de potencia aeróbica (Abdelkrim, Chaouachi, Chamari, Chtara, Castagna, 2010), potencia anaeróbica (Struzik, Pietraszewski, Zawadzki, 2014) y capacidad aeróbica (Apostolidis, Nassis, Bolatoglou, Geladas, 2004).

Desde el apartado de preparación física, el baloncesto es considerado un deporte intermitente de alta intensidad que requiere principalmente metabolismo anaeróbico (Castagna et al., 2010). Sin embargo, la duración del partido de baloncesto (40 minutos) requiere un alto nivel de metabolismo aeróbico con la finalidad de realzar la resíntesis de fosfocreatina, aclaramiento de lactato de la actividad muscular (Castagna et al., 2010).

Teniendo en cuenta lo anterior y pensando siempre en la mejora del rendimiento de jugadores, se debe plantear tareas de entrenamiento que tengan una trasferencia positiva sobre el juego. Por este motivo, es reseńable tener en cuenta los di- 
ferentes componentes de la carga de competición. A la hora de diseñar tanto la temporada como los entrenamientos que formarán dicho periodo de tiempo, hay que tener en cuenta la intensidad de cada tarea y la duración para así determinar la carga del entrenamiento.

De este modo, la carga del entrenamiento debe de seguir una serie de principios demostrados científicamente (principio de multilateralidad, especificidad, sistematización, incremento progresivo de la carga, continuidad, individualización y variedad entre otros) y que su correcta utilización provocan unos resultados positivos, por ello, "el proceso de planificación y control del entrenamiento es básico para conocer objetivamente como se realiza el entrenamiento en deportes colectivos" (Ibáñez, 2008). Por otro lado, Coque $(2008,2009)$ ańade que la cuantificación de la carga total del entrenamiento es una pieza clave, entendida ésta como una suma total de estímulos a los que el jugador se ve sometido durante el proceso de preparación o entrenamiento.

Aun conociendo dicha importancia, "los jugadores sometidos a importantes cargas de entrenamiento no disponen en muchas ocasiones del control de las mismas, ni del seguimiento, valoración de la adaptación y asimilación que estas provocan en su organismo. En la mayoría de ocasiones por el alto coste económico de los métodos tradicionales para la valoración del esfuerzo "(Del Campo, Álvarez y Lorenzo, 2008).

En el tema de la planificación, Sánchez (2007) afirma que "dependiendo del modo en que lo haga, la carga total del entrenamiento será transferible a un partido". Se puede conocer a través de la carga interna (Liberal y García-Mas, 2011; Molinero, Salguero y Márquez, 2011) y por otro lado mediante análisis de las escalas subjetivas de Coque (2008, 2009). Dicho proceso y control del entrenamiento es básico para conocer objetivamente cómo se realiza el entrenamiento en deportes colectivos.

En cuanto al modelo de enseńanza que se puede emplear a la hora de organizar y llevar a cabo las sesiones se puede encontrar multitud de corrientes o variantes. En esta investigación está basada en el enfrentamiento entre el modelo de enseńanza mas cercano al modelo tradicional, Allison y Thorpe (1997) "está basado en la habilidad y se caracterizan por la enseñanza de habilidades y técnicas específicas dentro de lecciones altamente estructuradas, con un formato que contiene un calentamiento, la práctica de unas destrezas y un juego final" y el modelo de enseñanza más próximo al modelo compresiva o alternativo “juegos deportivos para la comprensión, caracterizados por la conciencia táctica y la toma de decisiones dentro de la estructura de un juego deportivo apropiado, por el uso de juegos modificados y la enseñanza de habilidades cuando son apropiadas y siempre adaptadas a los niveles individuales" (Doolittle, 1995).

Estudios que comparen la condición física en diferentes deportistas se realizan en función de los puestos específicos y rol del jugador (Sánchez, 2007), del nivel de la liga (OliveiraDa-Silva, Sedano-Campo \& Redondo-Castán, 2013), de la edad (Binnetoğlu, Babaoğlu, Altun, Kayabey, 2014), del género (Vaquera, Villa, Morante, Renfree, Peters, 2016). Por este motivo, tras la revisión de la literatura no se encuentran estudios específicos que analicen la condición física de los deportistas en función del modelo de enseñanza llevado a cabo en los entrenamiento, por ello, el planteamiento del problema de esta investigación será analizar las diferencias existentes en la condición física en jugadores de baloncesto entrenados bajo dos modelos de enseńanza diferentes.

En cuanto a los objetivos de la investigación encontramos los siguientes:

A. Analizar el proceso de entrenamiento en el estudio de las Variables Pedagógicas, Variables de Carga Externa y Variables Organizativas.

B. Identificar que existen dos modelos de enseñanza diferentes utilizados en los entrenamientos analizados. De este objetivo se extrae la siguiente hipótesis:

I. El equipo bajo enfoque comprensivo utiliza de manera mas ordenadas y eficiente los diferentes entrenamientos en cuanto a las diferentes variables analizadas.

C. Analizar las diferencias en cuanto a los resultados obtenidos entre ambos equipos en las diferentes pruebas realizadas. De este objetivo se derivan a su vez en dos hipótesis:

I. El equipo bajo enfoque comprensivo obtiene mejores resultados en la prueba de Resistencia Aeróbica.

II. El equipo bajo enfoque comprensivo obtiene resultados similares al equipo bajo enfoque tradicional en cuanto a la prueba de Resistencia Anaeróbica.

\section{Método}

Para Ato, López y Benavente (2013) el modelo de la investigación se puede enmarcar bajo una estrategia manipulativa, dentro de los estudios de naturaleza empírica de tipo cuasiexperimental donde se busca examinar las diferencias entre grupos, Diseño de grupos no equivalentes.

La muestra de participantes seleccionadas para esta investigación han sido los equipos pertenecen al club San Antonio Cáceres Basket, de categoría Cadete que militan en la misma competición autonómica llamada Judex Plata. La selección de estos equipos se realizó debido a que los equipos realizan el mismo número de entrenamiento y con las mismas condiciones materiales. Además, el modelo de enseńanza utilizado en los entrenamiento que llevan a cabo sus entrenadores inicialmente es diferente. Este hecho será comprobado durante el desarrollo de esta investigación.

Las características que definen estos dos equipos son los siguientes:

Los sujetos del Cadete B, formado por 13 jugadores que tienen una edad media de $15.46 \pm 0.77$ ańos, una altura media de $178 \pm 8.70$ centímetros, una envergadura de $178 \pm 7.73$ cen- 
tímetros y un peso medio de $64.6 \pm 10.09$ kilogramos. Por el contrario, el Cadete $\mathrm{C}$, formado a su vez por 13 jugadores con una edad media de $14.23 \pm 0.46$ ańos, una altura media de 173 \pm 8.55 centímetros, una envergadura de $170 \pm 9.39$ centímetros y un peso medio de $61.5 \pm 9.30$ kilogramos. Cabe mencionar que el equipo Cadete $B$ es un equipo formado por la mitad de jugadores de primer año de la categoría y el resto formado por jugadores de segundo año de la categoría. Sin embargo, el Cadete $\mathrm{C}$ está formado íntegramente por jugadores de primer año de la categoría. Aunque existen diferencias en la edad, es la muestra mas pareja que se ha encontrado.
La muestra que forma la investigación se lleva a cabo duración de la segunda y definitiva parte del campeonato regional de Judex Plata en el que la primera jornada de dicha parte comienza la semana del 9 de Enero de 2016 y finaliza el 19 de Marzo de 2016.

En dicha muestra, los dos entrenadores que conforman la investigación realizan el Cuestionario COQ. En el gráfico 1 se observa una representación de los resultados que obtienen ambos entrenadores del Cadete B y Cadete $\mathrm{C}$ en el Cuestionario del Entrenador COQ.

Grafico 1. Representación gráfica del perfil de los entrenadores del cuestionario COQ.
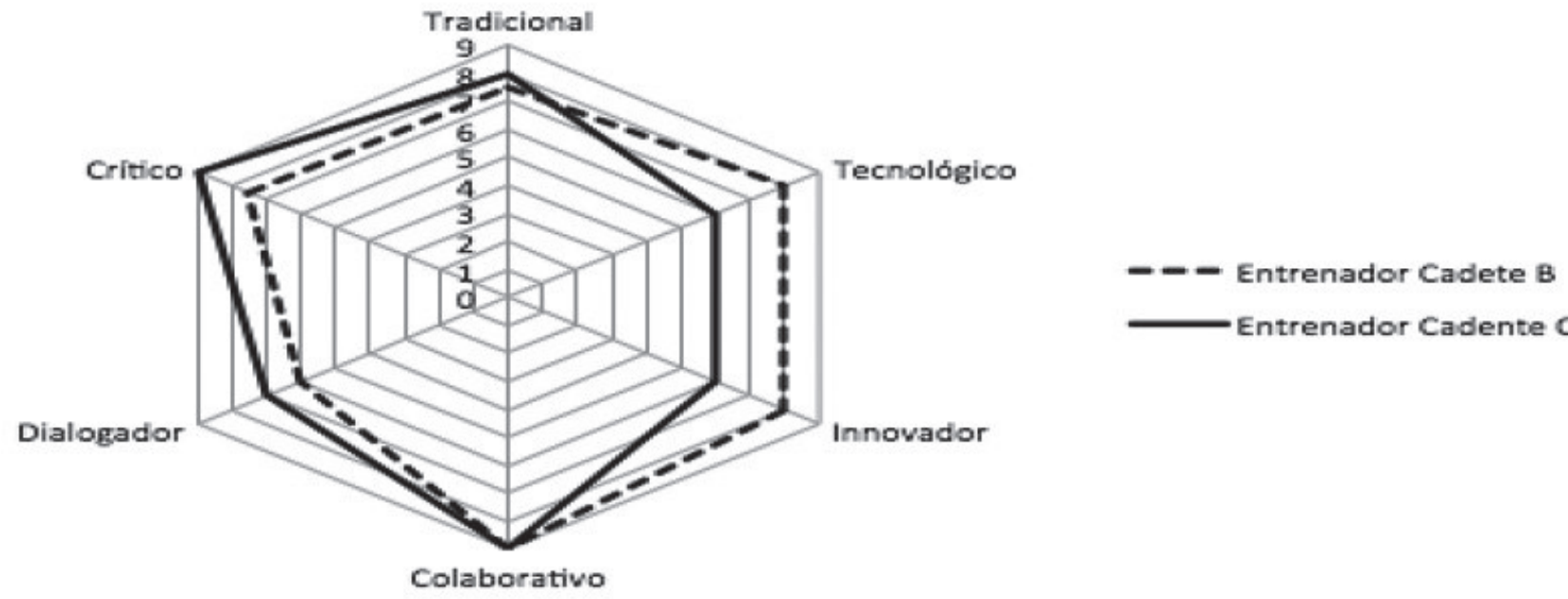

- Entrenador Cadente C

Como se observa, el entrenador del Cadete B, sus características mas destacables son tecnológico, innovador y colaborativo. Sin embargo, el entrenador Cadete $\mathrm{C}$ tiene como características principales tradicional, crítico y colaborativo. En este perfil ya se encuentra como un entrenador se cataloga así mismo como tradicional y otro entrenador como innovador, estos valores unidos al análisis de las sesiones nos hace tener una información completa de cada entrenador.

Para obtener mayor información sobre la muestra, se recogen las 230 tareas agrupadas en 28 sesiones para el equipo Cadete B y 205 tareas en 27 sesiones para el Cadete C. Durante este tiempo se llevan a cabo las diferentes pruebas de condición física para conocer el nivel aeróbico y anaeróbico de cada jugador.

Para mayor información sobre la muestra analizada, cada equipo realiza tres entrenamientos semanales (de lunes a viernes) con una duración de una hora y media cada entrenamiento. En cuanto a la organización de las sesiones por parte del equipo Cadete B, comienzan con situaciones individuales y a medida que avanza la sesión las tareas van volviéndose mas complejas para acabar con situaciones grupales o colec- tivas. Por parte del Cadete C, los entrenamientos estaban organizados en dos partes, la primera y de menor duración era dedicada a la preparación física en la que el preparador físico adaptaba los ejercicios para entrenar de manera integrada al deporte en la que se realizaban tareas de situaciones reducidas y una segunda parte del entrenamiento de mayor duración en la que el entrenador ya estaba a cargo del grupo y que se realizaban tareas grupales o colectivas.

En cuanto a la experiencia previa de los jugadores analizados es similar en ambos equipos y no existen diferencias.

\section{Variables del estudio}

Como variable independiente del estudio encontramos el proceso metodológico o modelo de enseñanza de cada entrenador que emplea a lo largo de los entrenamientos. Se lleva a cabo el análisis de las tareas de los entrenamientos desde las Variables Pedagógicas, Variables de Carga Externa y Variables Organizativas como recoge el Sistema Integral para el Análisis de las Tareas de Entrenamiento, SIATE, (Ibáñez, Feu, Cańadas, 2016). Además también se evalúa al entrenador a través 
del Cuestionario del Entrenador llamado Coach Orientation Questionnaire, COQ (Feu, Ibáñez, Graça, Sampaio, 2007). El COQ es un perfil teórico y una autodefinición del propio entrenador, por este motivo se busca una comparación entre el perfil teórico que tiene el entrenador de su autoimagen (lo que dice que hace) y lo que hace, intentamos comprobar si los entrenadores se autodefinen correctamente.

Las tareas fueron analizadas por un observador externo al cuerpo técnico de cada equipo y tras finalizar la recogida de tareas se realizó una reunión con cada cuerpo técnico en la que se buscaba la concordancia adecuada entre la planificación de la tarea y finalmente lo que se desarrollo. Los observadores eran expertos en el deporte de baloncesto, con el título de Graduado en Ciencias del Deporte y la Actividad Física y cuya especialidad deportiva fue el deporte del baloncesto.

Como variables dependientes de la investigación encontramos las variables relacionadas con el nivel de la resistencia Aeróbica y Anaeróbica que se ha medido.

\section{Instrumentos}

Para la realización de la investigación hemos utilizado lo siguiente:

Material: SIATE, reloj cronómetro, SPSS, dispositivos Wimü (dispositivo inalámbrico que controla la actividad física y que posee una tecnología de análisis cinemático, táctico y fisiológico), software Qüiko (Software del dispositivo Wimü).

Instrumentos: Test Aeróbico SIG/AER, Test Anaeróbico SIG/ANA y Cuestionario del Entrenador COQ (Coach Orientation Questionnaire).

El Test Aeróbico SIG/AER se caracteriza por ser una adaptación del test de Cooper al baloncesto, en esta prueba que tiene una duración de doce minutos, el jugador debe de intentar realizar el mayor número de vueltas al circuito. El circuito esta formado por doce partes, seis de ellas el jugador se desplaza en carrera con balón y finaliza realizando un tiro a distancia cercana, los otros 6 periodos del circuito, el jugador realiza desplazamiento sin balón entre lo que se encuentran desplazamientos de espalda, movimientos defensivos y sprint frontal. En esta prueba se intenta recopilar que el jugador realice movimientos y acciones que son específicas del deporte, lo que otorga a la prueba de mayor validez y especificidad.

El Test Anaeróbico SIG/ANA se basa en una adaptación del test aeróbico mencionado anteriormente, tiene una duración de 5 periodos cuyo periodo tiene una duración de 1 minuto y un descanso entre periodo de 1 minuto, durante el minuto del periodo el jugador debe realizar el mayor número de circuitos posible. Cada circuito está formado por 4 fragmento, en uno de ellos el jugador realiza desplazamiento sin balón, un periodo de carrera con balón y finaliza con una entrada, tercer periodo el jugador se desplaza de espalda y en el ultimo fragmento del circuito el jugador realiza movimientos defensivos.

\section{Procedimiento de aplicación}

La recogida de los diferentes entrenamientos y pruebas realizadas sobre la temporada 2015/2016 durante el período de Judex Plata (9 de Enero- 19 de Marzo) se realiza en el siguiente orden. A lo largo del periodo cada equipo se realiza el Test AER/SIG (Ibáñez, Sáenz-López, Gutiérrez; 1992a) de manera individual a todos los integrantes del equipo separados por un periodo de 1 minuto de diferencia entre componentes. Durante los 12 minutos que cada jugador se encuentra realizando la prueba, su finalidad será intentar dar el mayor número de vueltas al circuito. Este test trata de ser una alternativa a los test clásicos ya sean de campo o laboratorio. Además, al ser un test específico tiene conexión con el deporte y a su vez rigor científico.

Para la realización del Test ANA/SIG (Ibáñez, Sáenz-López, Gutiérrez; 1992b) que mide la resistencia anaeróbica láctica, se realiza de manera individual y consta de 5 periodos de trabajo con duración de un minuto por periodo y un minuto de descanso entre periodos.

Destacar que anterior a la realización de las pruebas físicas, los entrenadores realizaron el Cuestionario del Entrenador COQ para conocer sus perfiles y hacia que enfoque tiene mas proximidad. En cuanto a dicho Cuestionario, ambos entrenadores responden de manera personal e individual a una serie de preguntas que da información sobre diferentes perfiles referentes a los modelos que utiliza, la formación que tienen, la toma de decisión de los entrenadores y la planificación. Siendo las respuestas realizadas una autodefinición teórica de ellos mismos.

Durante el período de Judex Plata, se recogieron todas las tareas de los entrenamientos realizados de ambos equipos para así mediante las variables con las que interactúan en el entrenamiento cada equipo conocer si tienen relación con los resultados obtenidos en las pruebas de condición física realizadas.

Posteriormente, con ayuda del SIATE, se introducen los datos obtenidos de la observación de las tareas. Seguidamente, se realiza una reunión con el cuerpo técnico de cada equipo para presentar los datos tabulados y comparar la concordancia y corregir posibles erratas.

Por último, con ayuda del software estadística SPSS se realiza una serie de análisis para interpretar los datos obtenidos en el análisis y poder dar unos resultados objetivos.

\section{Análisis estadístico}

En este apartado se realizó en primer lugar un análisis exploratorio de los datos obtenidos, en segundo lugar, un análisis descriptivo para obtener información sobre cantidad de veces que se ha producido una variable en concreto (frecuencia y $\%$ para variables cualitativas así como Media y Desviación 
Típica para las cuantitativas). En tercer lugar se realizó el análisis inferencial que son las pruebas de asunción de criterios formado por las pruebas del Supuesto de Normalidad (Prueba de Shapiro Wilk), de contraste del Supuesto de Homocedasticidad (Prueba de Levene), de contraste del Supuesto de Aleatorización (Prueba de Rachas), para establecer una contrastación del modelo de la hipótesis adecuada (Field, 2009). Además, se realizó la prueba MANOVA y se utilizo el valor de significación del estadístico $F$ y los grados de libertad. Se acompañó a los niveles de significación de la magnitud del efecto alcanzado y la potencia estadística del diseño, lo que permite al lector comprender adecuadamente y de manera mas completa los resultados del análisis constituyendo una prueba mas relevante para la validez de dicho estudio (Cárdenas Castro y Arancibia, 2014).

\section{Resultados}

Como se observa en la tabla 1 , los resultados descriptivos que comparan las sesiones analizadas en cuanto a las variables del instrumento SIATE. En esta tabla encontramos el número de veces que se realiza cada categoría dentro de la misma variable y el porcentaje que representa del total de los ejercicios.

Tabla 1. Resultados descriptivos de las Variables Pedagógicas de las sesiones.

\begin{tabular}{|c|c|c|c|c|c|}
\hline & & \multicolumn{2}{|c|}{ CADETE C } & \multicolumn{2}{|c|}{ CADETE B } \\
\hline & VARIABLE & $n$ & $\%$ & $n$ & $\%$ \\
\hline Situación & SSG & 145 & 70 & 197 & 85,65 \\
\hline De Juego & FG & 60 & 30 & 33 & 14,35 \\
\hline Fase & Ataque & 105 & 51.22 & 116 & 54,8 \\
\hline De & Defensa & 35 & 17.07 & 46 & 20 \\
\hline \multirow[t]{2}{*}{ Juego } & Mixta & 47 & 22.93 & 26 & 11,3 \\
\hline & Prep. Física & 18 & 8.78 & 5 & 2,1 \\
\hline Tipo & Conductas TT & 102 & 49.76 & 130 & 57.00 \\
\hline De & Gestos TT & 63 & 30.73 & 72 & 31.00 \\
\hline \multirow[t]{2}{*}{ Contenido } & $\begin{array}{l}\text { Calentamiento/ } \\
\text { Estiramientos }\end{array}$ & 40 & 19.51 & 28 & 12.00 \\
\hline & EA & 130 & 63.42 & 7 & 0,9 \\
\hline \multirow{5}{*}{ Medio } & JE & 28 & 13.65 & 181 & 78.8 \\
\hline & Predeporte & 0 & 0 & 22 & 9,6 \\
\hline & Deporte & 47 & 22.93 & 25 & 10,5 \\
\hline & Sin Oposición & 121 & 59.03 & 85 & 37 \\
\hline & Oposición & 84 & 40.97 & 139 & 60,4 \\
\hline \multirow[t]{3}{*}{ Oposición } & Opos. Modulada & 0 & 0 & 6 & 2,6 \\
\hline & Simultaneo & 85 & 41.46 & 149 & 64,8 \\
\hline & Alternativo & 95 & 46.35 & 45 & 19,6 \\
\hline Participación & Consecutivo & 25 & 12.19 & 36 & 15,7 \\
\hline
\end{tabular}

En cuanto a las mayores diferencias existentes entre equipos se destacan en la variable Situación de Juego donde Small Sided Games (SSG) que encuadramos las situaciones de entrenamiento desde el $1 \mathrm{x} 0$ contra el $3 \mathrm{x} 3$ y las situaciones de Full Games (FG) formadas por las situaciones de 4x4 y 5x5.

En cuanto a las variables Fases de Juego, las opciones de ejercicios con finalidad ataque, defensa, mixto o con la finalidad de preparación física sufren pequeñas diferencias entre equipos.

La variable Tipo de Contenido también muestra pequeñas diferencias entre los ejercicios que se encuentran catalogados como Conductas Técnico- Tácticas (Conductas TT) y Gestos Técnico- Tácticos (Gestos TT).

También se encuentran grandes diferencias en la variable Medio dónde el equipo Cadete $\mathrm{C}$ realiza mayoritariamente de la opción Ejercicio de Aplicación (EA) mientras que el reparto en el resto de opciones es menor. Por el contrario, el Cadete $\mathrm{B}$ dedica mayor número de ocasiones a tareas encuadradas como Juegos Específicos (JE). Estas diferencias encontradas también es in indicador que confirman las diferencias en los modelos de entrenamiento a la hora de la programación del entrenamiento y la metodología que se lleva a cabo, por este motivo, la diferencia en una variable no explica un modelo u otro pero la suma de diferencias provoca que estos modelos sean diferentes. Cabe destacar por último, que las variables Predeporte (situaciones de $4 \times 4)$ y Deporte $(5 \times 5)$ también sufren diferencias entre equipos aunque menores que las anteriormente citadas.

Se observa que la variable Oposición también muestra diferencias entre equipos y entre tareas que se realizan sin oposición y tareas que se realizan con alguna oposición.

Por último, en la variable Organización también encontramos diferencias en cuanto a las opciones elegidas y los equipos que las realizan.

En la tabla 2, se encuentra a continuación los valores de cada variable cualitativa en cuanto a la prueba de $U$ de Mann Whitney. Además se realiza la potencia estadística y el tamaño del efecto.

Se observa en esta tabla que todas las variables de la tabla son significativas a excepción de la variable Participación.

Los resultados que se observan pertenecientes a las tareas analizadas confirman que existen diferencias entre las distintas metodologías de entrenamiento empleadas. En cuanto a la potencia estadística se encuentran valores óptimos que suponen diferencias significativas $(>.80)$ en todas las variables exceptuando la variable Participación. En cuanto a la prueba del tamaño del efecto, según Cárdenas y Arancibia (2014) en el caso de la variable Carga Competitiva se valora un tamańo del efecto grande (>.40) mientras que en el resto de variables analizadas el valor se encuentra en un tamaño mediano $(>.25)$ y pequeño $(>.10)$. 
Tabla 2. Resultados inferenciales de variables cualitativas del proceso de entrenamiento.

\begin{tabular}{|c|c|c|c|c|c|c|c|c|c|c|}
\hline & Ude Mann Whitney & $p$ & & $F$ & $g l 1$ & $g l 2$ & $p$ & & $\eta 2$ & $\phi$ \\
\hline Situación de Juego & 24995.00 & .000 & $*$ & 19.858 & 11 & 392 & .000 & $*$ & .338 & 1.000 \\
\hline Fase de Juego & 16824.00 & .003 & $*$ & 7.432 & 5 & 398 & .000 & $*$ & .085 & 999 \\
\hline Tipo de Contenido & 16786.50 & .005 & $*$ & 5.358 & 12 & 391 & .000 & $*$ & .141 & 1.000 \\
\hline Medio & 18321.00 & .073 & & 10.740 & 5 & 398 & .000 & $*$ & .119 & 1.000 \\
\hline Nivel de Oposición & 22525.00 & .009 & $*$ & 6.049 & 2 & 400 & .003 & $*$ & .029 & .883 \\
\hline Grado de Opos. & 21419.50 & .183 & & 16.861 & 2 & 401 & .000 & $*$ & .078 & 1.000 \\
\hline Densidad & 19828.00 & .870 & & 32.985 & 4 & 399 & .000 & $*$ & .249 & 1.000 \\
\hline Núm. eje. Simult. & 15430.50 & .000 & $*$ & 26.997 & 3 & 400 & .000 & $*$ & .168 & 1.000 \\
\hline Carga Competitiva & 21172.00 & .286 & & 69.481 & 4 & 399 & .000 & $*$ & .411 & 1.000 \\
\hline Espacio de Juego & 16558.00 & .002 & $*$ & 36.762 & 4 & 399 & .000 & $*$ & .269 & 1.000 \\
\hline Implicación Cogn. & 23884.00 & .000 & $*$ & 46.648 & 4 & 399 & .000 & $*$ & .319 & 1.000 \\
\hline Participación & 19586.00 & .670 & & .799 & 2 & 401 & .450 & & .004 & .186 \\
\hline
\end{tabular}

En la tabla 3, mostrada a continuación, se observan las variables cuantitativas que tienen relación con las tareas de entrenamiento analizadas. Se puede observar que en todas las variables que se muestran en la tabla existe diferencia significativa y que la potencia estadística es óptima (>.80). Sin embargo, en cuanto a la prueba que se realizó para conocer el tamaño del efecto, en esta ocasión todas las variables tienen un valor alto $(>.40)$, esto provoca que los resultados además de tener diferencias significativas, corrobora las diferencias que se encuentran entre ambas metodologías en cuanto a sus entrenamientos.

Tabla 3. Resultados inferenciales de variables cuantitativas del proceso de entrenamiento.

\begin{tabular}{lcccccccccccc}
\hline \multicolumn{10}{c}{ Cadete C } & \multicolumn{1}{c}{ Cadete B } \\
\hline Variable & Media & SD & Media & $S D$ & $F$ & gll & gl2 & $p$ & & $\eta 2$ & $\phi$ \\
\hline Carga Total & 18.64 & 7.963 & 17.89 & 6.38 & 31.651 & 22 & 381 & .000 & $*$ & .646 & 1.000 \\
Carga Total Seg. & 649.46 & 251.72 & 501.21 & 260.93 & 2.703 & 214 & 189 & .000 & $*$ & .754 & 1.000 \\
Carga Total Min. & 10.828 & 4.19 & 8.35 & 4.349 & 1.738 & 127 & 276 & .000 & $*$ & .444 & 1.000 \\
Tiempo Total & 870.89 & 471.81 & 545.27 & 280.16 & 3.385 & 200 & 203 & .000 & $*$ & .769 & 1.000 \\
Tiempo Expl. & 221.55 & 249.11 & 48.48 & 37.87 & 5.618 & 119 & 263 & .000 & $*$ & .718 & 1.000 \\
Tiempo Útil & 649.34 & 251.79 & 501.21 & 260.93 & 2.661 & 214 & 189 & .000 & $*$ & .751 & 1.000 \\
Aprovechamiento & 81.05 & 14.18 & 92.17 & 5.70 & 2.358 & 279 & 124 & .000 & $*$ & .841 & 1.000 \\
\hline
\end{tabular}
$p<.05$

Por último, en la tabla 4, se realiza una comparación des- ción física (Resistencia Aeróbica y Anaeróbica). criptiva de los resultados obtenidos en las pruebas de condi-

Tabla 4. Resultados descriptivos e Inferenciales pruebas de condición física.

\begin{tabular}{lccccccccccc}
\hline Variables & \multicolumn{1}{c}{ Tradicional } & \multicolumn{1}{c}{ Comprensivo } & \multicolumn{1}{c}{} \\
\hline & Media & $S D$ & Media & $S D$ & $F$ & gl & gl2 & $p$ & & $\eta 2$ & $\phi$ \\
\hline No de Circuitos Aer. & 134.36 & 10.37 & 146.54 & 9.80 & 8.722 & 1 & 22 & .007 & $*$ & .284 & .806 \\
No de Lanzamientos & 11.36 & .924 & 12.23 & .927 & 5.228 & 1 & 22 & .032 & $*$ & .192 & .589 \\
Aciertos & 6.09 & 2.81 & 6.08 & 2.29 & .000 & 1 & 22 & .989 & .000 & .050 \\
\% Acierto & 41.52 & 27.35 & 49.46 & 18.30 & .293 & 1 & 22 & .593 & .012 & .082 \\
FC Media & 183 & 4.80 & 186.13 & 8.00 & .980 & 1 & 22 & .338 & .061 & .153 \\
FC Máxima & 194 & 5.39 & 197.63 & 7.46 & 1.342 & 1 & 22 & .265 & .082 & .192 \\
\hline
\end{tabular}




\begin{tabular}{|c|c|c|c|c|c|c|c|c|c|c|c|}
\hline \multirow[t]{2}{*}{ Variables } & \multicolumn{2}{|c|}{ Tradicional } & \multicolumn{2}{|c|}{ Comprensivo } & \multirow[b]{2}{*}{$F$} & \multirow[b]{2}{*}{$g l 1$} & \multirow[b]{2}{*}{$g l 2$} & \multirow[b]{2}{*}{$p$} & & \multirow[b]{2}{*}{$\eta 2$} & \multirow[b]{2}{*}{$\phi$} \\
\hline & Media & $S D$ & Media & $S D$ & & & & & & & \\
\hline No de Circuitos Ana. & 94.86 & 10.76 & 110 & 7.00 & 13.912 & 1 & 22 & .001 & $*$ & .387 & .945 \\
\hline Aciertos Total & 12.45 & 10.27 & 22.92 & 3.32 & 12.075 & 1 & 22 & .002 & * & .354 & .913 \\
\hline$\%$ Acierto Total & 51.70 & 41.93 & 81.76 & 10.61 & 6.256 & 1 & 22 & .020 & * & .221 & .667 \\
\hline FC Media Total & 119.03 & 94.60 & 132.28 & 91.89 & .120 & 1 & 22 & .005 & $*$ & .120 & .063 \\
\hline
\end{tabular}

Por parte de la prueba Aeróbica, tan sólo encontramos como variables significativas el Número de Circuitos y el Número de Lanzamientos (directamente relacionados). En estas variables se encuentra que el equipo más próximo al enfoque comprensivo recorre mayor número de fracciones de circuitos (lo que lleva a afirmar que recorre mayor distancia) y que por lo tanto realiza mayor número de lanzamientos a canasta. En cuanto a estas variables la potencia estadística se encuentra en valores óptimos (>.80) aunque el tamaño del efecto es mediano para la primera variable y pequeńo para la segunda.

En las variables Anaeróbicas, todas las variables analizadas son significativas y a excepción de la FC Media Total, la potencia estadística es óptima o media- alta en su defecto. Sin embargo, en cuanto al tamaño del efecto, las variables tienen un tamaño pequeño (FC Media Total y \% de Acierto Total) y medio (Número de Circuitos Anaeróbicos y Aciertos Totales), lo que confirma las diferencias encontradas entre equipos y que el equipo cercano al enfoque comprensivo también recorre mayor distancia en el mismo periodo de tiempo y trabajando con Frecuencias Cardíacas similares al equipo próximo al enfoque tradicional.

\section{Discusión}

El objetivo del presente estudio ha sido determinar que modelo de enseńanza de los empleados que son comparadas es mas óptimo para favorecer y potenciar tanto la resistencia aeróbica como anaeróbica de dichos jugadores.

Para Casamichana, Castellano, González-Morán, GarcíaCueto, García- López, (2011) en los deportes de equipo, desde hace algunas décadas una alternativa al entrenamiento tradicional sin balón han sido los juegos reducidos. Estas formas jugadas son tareas de entrenamiento con los rasgos de un duelo colectivo. Son situaciones utilizadas habitualmente como medio de entrenamiento (Ford, Yates, y Williams, 2010) en el que las dimensiones del campo, el número de jugadores y las reglas que rigen el mismo se modifican con el fin de conseguir unos objetivos determinados ya sean técnicos, tácticos o físicos. En el caso de esta investigación se puede observar que las situaciones reducidas (SSG) en ambos equipos son mas utilizadas que las situaciones de $4 \times 4$ o $5 \times 5$ (FG).

En la actualidad, es más frecuente la utilización de este tipo de situaciones de entrenamiento con el objetivo de desarrollar la capacidad condicional del deportista, mostrándose como un método tan efectivo como el entrenamiento interválico (Hill-Haas, Coutts, Rowsell, y Dawson, 2009). Este método alternativo tiene la ventaja de que permite trabajar a la vez aspectos técnico-tácticos y físicos, aportando al entrenamiento una mayor especificidad y una optimización del tiempo de entrenamiento. McCormick, Hannon, Newton, Shultz, Miller, Young (2012), compararon situaciones de 3x3 y $5 \times 5$, establecieron que no existían diferencias significativas entre tareas de Intensidad Moderada y tareas de Intensidad Vigorosa. A su vez, Duarte, Batalha, Folgado, Sampaio, (2009) concluyeron que en situaciones de $2 \times 2$, 3x3 y 4x4, la situación de $4 \times 4$ es donde la frecuencia cardíaca alcanza su porcentaje más bajo. Corroborando los datos del estudio tanto para la categoría infantil como para la cadete.

En cuanto a los aspectos físicos, Coque (2008, 2009) en las variables pertenecientes a la Carga Externa de las sesiones analizadas afirma que no debe existir gran variedad en cuanto al trabajo físico realizado ya que esta variable debe mantenerse presente durante toda la temporada. Siguiendo en esta línea Halouani, Chtourou, Gabbett, Chaouachi, Chamari, (2014) confirma que algunas modificaciones relacionadas con el número de jugadores en las tareas que forman parte de la Carga Externa son producidas por las adaptaciones fisiológicas del jugador y se demostró que las situaciones de 3x3 en fútbol obtuvo mayor intensidad que la variante $5 \times 5$. Otra afirmación es la realizada por Hill-Haas, Dawson, Coutts, Rowsell, (2009) que explica que hay que tener en cuenta las agrupaciones en las tareas, a medida que avanza la temporada las agrupaciones tienden a ser de mayor número, lo que provoca mayor variación tanto fisiológica como perceptivamente. En este caso, el equipo Cadete $\mathrm{B}$ tiene mayor número en situaciones de juego de Small Sites Games (SSG), mientras que el Cadete $\mathrm{C}$ obtiene mayor resultado en situación de Full Game (FG). En este caso, al utilizar escalas subjetivas para conocer la carga externa de la tarea, si existen diferencias provocadas por la variación de agrupaciones en las tareas de entrenamiento.

Siguiendo las variables que tienen relación con las tareas analizadas, Alarcón, Cárdenas y Ureña (2008) habla de la Participación de las tareas en las que a mayor valor, mayor aprovechamiento del tiempo de entrenamiento. En cuanto al Cadete $\mathrm{B}$ el aprovechamiento medio de las tareas es del 92.17\% Mientras que el Cadete $\mathrm{C}$ tiene un $81.05 \%$ en sus tareas analizadas. Ambos resultados se encuentran por encima 
del $80 \%$ que recomienda el autor, aunque existen diferencias entre ambos entrenadores analizados. Continuando en esta variable, Alarcón et al., (2008) confirman que en la participación simultánea obtiene un $60 \%$ de tiempo de ejecución, $30 \%$ en alternativas y por último $10 \%$ en tareas con participación consecutivas. Los autores mencionados anteriormente también confirman que si se trabaja bajo una organización mayoritariamente simultánea, los jugadores realizan mayor número de vivencias en la tarea al existir el tiempo de espera menor, además de una mejora en cuanto a las cualidades, esta mejora será mayor que si el entrenador elige otro tipo de organización para las tareas. Si comparamos con los resultados obtenidos, el Cadete $\mathrm{B}$ obtiene mayor número de tareas simultáneas, mientras que el Cadete $\mathrm{C}$ obtiene mayor número en tareas de tipo alternativo. En cuanto a la distribución de intensidades de trabajo fisiológico, encontramos cómo los deportistas del equipo bajo enfoque comprensivo tiene mayor aprovechamiento del tiempo de entrenamiento, lo que corrobora que su trabajo es mas intenso y con menor descanso, provocando mejores resultados aeróbicos.

En los enfoques tradicionales, la estructura típica de sesión acarrea mayores problemas con respecto a los enfoques alternativos, debido a que convierten en eje del proceso de enseñanza al contenido, mientras que los enfoques alternativos sitúan al jugador como centro sobre el que debe girar la enseñanza (Thorpe y Bunker, 1983).

Los enfoques tradicionales comienza con una actividad introductoria, seguida de una práctica de la habilidad y al final un juego en el que se practicaba la nueva habilidad adquirida (Thorpe y Bunker, 1983).En este caso, el equipo Cadete C tiende a realizar mayor número de Ejercicios de Aplicación (EA), mientras que el Cadete $\mathrm{B}$ obtiene mayor resultados en tareas enmarcadas como Juegos Complejos (JC). Sin embargo, los enfoques alternativos están basados sobre una forma estándar de juego deportivo que es modificada poco a poco para ayudar al jugador a encauzar importantes conceptos y estrategias de todos los deportes (Doolittle, 1995). El juego se convierte en el elemento clave del enfoque comprensivo, haciéndole ver al niño el ejercicio como una actividad lúdica deportiva, más que como una práctica de habilidad determinada por el profesor que prohíbe u obstruye la práctica deportiva.

En cuanto a los resultados obtenidos en las pruebas de condición física, Sallet, Perrier, Ferret, Vitelli, Baverel, (2005) efectuaron un estudio con jugadores franceses que confirman que la capacidad anaeróbica puede considerarse uno de los factores de rendimiento más importantes en esta modalidad deportiva, independientemente de que cuantitativamente la vía aeróbica goce de una mayor importancia en el suministro energético.

Como ya se ha señalado, los resultados obtenidos confirman la naturaleza híbrida de este deporte en el que se alter- nan esfuerzos aeróbicos y anaeróbicos. En este sentido, las actividades predominantes durante un partido tienen carácter aeróbico (parado/caminando, trote, carrera de baja y media intensidad), ocupando éstas un porcentaje total aproximado del $85 \%$, mientras que las actividades que pudiéramos considerar de alta intensidad o anaeróbicas (sprint de alta intensidad y salto) ocupan el 15\% restante. Esta naturaleza híbrida ya ha sido mencionada por otros autores en estudios anteriores efectuados con muestras de jugadores jóvenes (Abdelkrim, El Fazaa, El Ati, 2007) y jugadores adultos pero no de élite (Narazaki, Berg, Stergiou, Chen, 2009).

El hecho de que esas acciones de carácter anaeróbico ocupen un menor porcentaje del tiempo real de juego no quiere decir que sean menos importantes, puesto que tal y como se ha señalado (Tessitore, Meeusen, Piacentini, Demarie, Capranica, 2006) son determinantes en situaciones vitales para el rendimiento como los contraataques, la recuperación defensiva, los lanzamientos o las acciones individuales de ataque frente a la canasta.

Abdelkrim et al. (2007) y Narazaki et al. (2009) afirman que ese patrón intermitente al que se hace referencia tiene un reflejo claro en la carga fisiológica y metabólica a la que se ve sometido el jugador durante la competición, según se extrae de los datos relativos a frecuencia cardiaca media, a acumulación de lactato o a consumo de oxígeno. La alta acumulación de lactato así como la elevada frecuencia cardiaca media pone en evidencia la importante demanda fisiológica impuesta sobre los jugadores de baloncesto durante la competición, independientemente de que el porcentaje de tiempo ocupado por las actividades de alta intensidad sea pequeño. En la misma línea, Narazaki et al. (2009) afirman que el baloncesto competitivo requiere de una importante utilización del metabolismo aeróbico, pero son los fosfágenos la fuente energética fundamental en esas acciones cortas de alta intensidad. Dicha vía metabólica tiene que ser restaurada continuamente durante la práctica, cobrando especial importancia en este sentido la vía aeróbica. Por su parte, Sallet et al., (2005) indican que, si bien existe bastante homogeneidad en las demandas aeróbicas al comparar distintos niveles competitivos, la capacidad anaeróbica parece ser un mejor predictor del rendimiento.

Como datos destacables, podemos afirmar que el equipo Cadete $\mathrm{B}$ tiene mejor resistencia aeróbica cuyo resultados informan que es mayor distancia la que recorre y mayor posibilidad de lanzamiento. Sin embargo, las frecuencias cardíacas de trabajo durante la prueba es similar en ambos equipos, por este motivo, trabajando en valores similares, el equipo Cadete $\mathrm{B}$ obtiene mayor rendimiento.

En cuanto a la resistencia anaeróbica, el equipo Cadete B obtiene mejores resultados derivados de la mayor distancia que recorren y mayor posibilidad de lanzamiento aunque en este caso si existen diferencias en cuanto a la frecuencia car- 
díaca de trabajo debido a la variable de frecuencia cardíaca en recuperación que existen diferencias entre equipos y que el equipo mencionado anteriormente realiza de manera mas eficiente y rápida que el equipo rival.

\section{Agradecimientos}

Este trabajo ha sido parcialmente subvencionado por la Ayuda a los Grupos de Investigación (GR15122) de la Junta de Extremadura (Consejería de Economía e Infraestructuras); con la aportación de la Unión Europea a través de FEDER

\section{Bibliografía}

1. Abdelkrim, B.N., El Fazaa, S., \& El Ati, J. (2007). Time-motion analysis and physiological data of elite under-19 basketball players during competition. British Journal of Sports Medicine, 41, 69-75.

2. Abdelkrim, B.N., Chaouachi, A., Chamari, K., Chtara, M., \& Castagna C. (2010) Positional role and competitive-level differences in elite-level men's basketball players. Journal of Strength and Conditioning Research, 24, 1346-1355.

3. Alarcón, F., Cárdenas, D \& Ureña, N. (2008). Influencia de los factores de organización de las tareas de aprendizaje sobre los tiempos de práctica del jugador de baloncesto. Apunts, Educación Física y Deportes 92, 46-55.

4. Allison, S. y Thorpe, R. (1997). A Comparison of the Effectiveness of two approaches to Teaching Games within Physical Education. A Skills approach versus a Games for Under- standing approach. The British Journal of Physical Education 28(3), 9-13.

5. Apostolidis N., Nassis G.P., Bolatoglou, T., Geladas, N.D. (2004) Physiological and technical characteristics of elite young basketball players. Journal Sports Medicine Physical Fitness, 44, 157-163.

6. Ato, M., López, J. J., \& Benavente, A. (2013). Un sistema de clasificación de los diseños de investigación en psicología. Anales de Psicologia, 29(3), 1038-1059.

7. Binnetoğlu, F. K., Babaoğlu, K., Altun, G., \& Kayabey, Ö. (2014). Effects that different types of sports have on the hearts of children and adolescents and the value of two-dimensional strain-strain-rate echocardiography. Pediatric cardiology, 35(1), 126-139.

8. Casamichana, D., Castellano, J., González-Morán, A., García-Cueto, H., \& García-López, J. (2011). Demanda fisiológica en juegos reducidos de fútbol con diferente orientación del espacio. RICYDE. Revista Internacional de Ciencias del Deporte, 7(23).

9. Castagna, C., Manzi, V., Impellizzeri, F., Chaouachi, A., Abdelkrim, B.N., \& Ditroilo, M. (2010). Validity of an on-court lactate threshold test in young basketball players. Journal of Strength Conditioning Research, 24, 2434-2439.

10. Cárdenas Castro, M., \& Arancibia Martini, H. (2014). Potencia estadística y cálculo del tamańo del efecto en $G^{*}$ Power: complementos a las pruebas de significación estadística y su aplicación en psicología. Statistical power and effect size calculating in $G^{*}$ Power. Salud \& Sociedad, 5(2), 210-224.

11. Chaouachi, A., Brughelli, M., Chamari, K., Levin, G.T., Abdelkrim, N., Laurencelle, L., \& Castagna, C. (2009). Lower limb maximal dynamic strength and agility determinants in elite basketball players. Journal of Strength and Conditioning Research, 23, 1570- 1577.

12. Coque, I. (2008). Valoración subjetiva de la carga del entrenamiento técnico-táctico. Una aplicación práctica (I). Clinic, 81, 39-43.

13. Coque, I. (2009). Valoración subjetiva de la carga del entrenamiento técnico-táctico. Una aplicación práctica (II). Clinic, 82, 42-45.

14. Del Campo, J., Álvarez, J., \& Lorenzo, A. (2008). La percepción del esfuerzo: concepto, características y aplicación al control del entrenamiento en baloncesto. En: Terrados, N., y Calleja, J. (eds.). Fisiología, Entrenamiento y Medicina del Baloncesto. 1a edición. (pp. 121-134) Badalona: Paidotribo.

15. Doolittle, S. (1995). Teaching Net Games to Low - Skilled Students: A Teaching for Understanding Approach. The Journal of Physical Educa- tion, Recreation \& Dance; 66(7), 18-23.

16. Duarte, R., Batalha, N., Folgado, H., \& Sampaio, J. (2009). Effects of exercise duration and number of players in heart rate responses and technical skills during futsal small-sided games. The Open Sports Sciences Journal, 2, 1-5.

17. Feu, S., Ibáñez, S. J., Graça, A., \& Sampaio, J. (2007). Evaluación psicométrica del cuestionario de orientación de los entrenadores en una muestra de entrenadores españoles de balonmano. Psicothema, 19(4), 699-705.

18. Field, A. (2009). Discovering statistics using SPSS. Third Edition. Londres: SAGE.

19. Ford, P. R., Yates, I., \& Williams, A. M. (2010). An analysis of practice activities and instructional behaviours used by youth soccer coaches during practice: exploring the link between science and application. Journal of Sports Sciences, 28(5), 483-495.

20. Halouani, J., Chtourou, H., Gabbett, T., Chaouachi, A., \& Chamari, K. (2014). Small-sided games in team sports training: A brief review. The Journal of Strength Conditioning Research; 28(12): 3594-3618.

21. Hill-Haas, S., Dawson, B., Coutts, A., \& Rowsell, G. (2009). Physiological responses and time-motion characteristics of various small-sided soccer games in youth players. Journal of Sports Sciences, 27(1), 1-8.

22. Ibáńez, S.J., Sáenz-López, P., \& Gutiérrez, A. (1992a) Test sig/aer, aeróbico específico sobre el terreno, para jugadores de baloncesto. In Congreso cientifico olímpico. Unisport/Junta de Andalucía. Fis-22.

23. Ibáńez, S.J., Sáenz-López, P., \& Gutiérrez, A. (1992b). Test sig/ana, anaeróbico específico sobre el terreno, para jugadores de baloncesto. InCongreso cientifico olimpico. Unisport/Junta de Andalucia. Fis-22.

24. Ibáñez, S. J. (2008). La planificación y el control del entrenamiento técnico-táctico en baloncesto. En N. Terrados \& J. Calleja (Coord.), Fisiología, entrenamiento y medicina del baloncesto (pp. 299-313). Barcelona: Editorial Paidotribo.

25. Ibáñez, S. J., Feu, S., \& Cañadas, M. (2016). Sistema integral para el análisis de las tareas de entrenamiento, SIATE, en deportes de invasión. E-balonmano. com: Revista de Ciencias del Deporte, 12(1), 3-30.

26. Liberal, R. \& García-Mas, A. (2011). Percepción de dolor y fatiga en relación con el estado de ánimo. Cuadernos de Psicología del Deporte, 11(2), 93-117.

27. McCormick, B. T., Hannon, J. C., Newton, M., Shultz, B., Miller, N., \& Young W. (2012). Comparison of Physical Activity in small-sided basketball games versus full-sided games. International Journal of Sports Science and Coaching, 7(4), 689-697.

28. Molinero, O., Salguero, A. \& Márquez, S. (2011). Análisis de la recuperación-estrés en deportistas y relación con los estados de ánimo: Un estudio descriptivo. Cuadernos de Psicología del Deporte, 11(2), 47-55.

29. Narazaki, K., Berg, K., Stergiou, N., \& Chen, B. (2009). Physiological demands of competitive basketball. Scandinavian Journal of Medicine and Science in Sports, 19, 425-432.

30. Oliveira-Da-Silva, L., Sedano-Campo, S., \& Redondo-Castán, J. C. (2013). Características del esfuerzo en competición en jugadoras de baloncesto de élite durante las fases finales de la Euroliga y el Campeonato del Mundo. RICYDE. Revista Internacional de Ciencias del Deporte, $9(34), 360-376$. 
31. Sallet, P., Perrier, D., Ferret, J.M., Vitelli ,V., \& Baverel, G. (2005). Physiological differences in professional basketball players as a function of playing position and level of play. The Journal of Sports Medicine and Physical Fitness, 45, 291-294.

32. Sánchez, M. S. (2007). El acondicionamiento físico en baloncesto. Apunts. Medicina de l'Esport, 42(154), 99-107.

33. Struzik, A., Pietraszewski, B., \& Zawadzki, J. (2014). Biomechanical Analysis of the Jump Shot in Basketball. Journal of Human Kinetics, 42, 73-79.

34. Tessitore, A., Tiberi, M., Cortis, C., Rapisarda, E., Meeusen, R., \& Capranica, L. (2006). Aerobic-anaerobic profiles, heart rate and match analysis in old basketball players. Gerontology, 52, 214-222.
35. Thorpe, R., \& Bunker, D. (1983). A new approach to the teaching of games in physi- cal education curriculum. En VV.AA. Teaching Team Sports (229-238). Roma: Congreso AIESEP.

36. Vaquera, A., Villa, J. G., Morante, J. C., Thomas, G., Renfree, A. J., \& Peters, D. M. (2016). Validity and Test-Retest Reliability of the TIVRE-Basket Test for the Determination of Aerobic Power in Elite Male Basketball Players. The Journal of Strength \& Conditioning Research, 30(2), 584-587.

37. Ziv, G., \& Lidor, R. (2009). Vertical jump in female and male basketball players-A review of observational and experimental studies. Journal of Science and Medicine in Sports, 13(3), 332-9. 